\title{
Loop-Mediated Isothermal Amplification Assay Targeting the 47-Kda Gene of Orientia tsutsugamushi: A Rapid and Sensitive Alternative to Real-Time PCR
}

\author{
Erin Huber ${ }^{1,2}$, Darder $\mathrm{Ji}^{3}$, Lee Howell ${ }^{1,2}$, Zhiwen Zhang ${ }^{1,2}$, Hua-Wei Chen ${ }^{1,2}$, Wei-Mei Ching ${ }^{1,2}$, Chien-Chung Chao ${ }^{1,2}$, \\ ${ }^{1}$ Viral and Rickettsial Diseases Department, Infectious Diseases Directorate, Naval Medical Research Center, Silver Spring, MD, USA \\ 2Uniformed Services University of the Health Sciences, Bethesda, MD, USA \\ ${ }^{3}$ Research and Diagnostic Center, Centers for Disease Control, Department of Health, 161 Kun-Yang Street, Taipei 11579, Taiwan
}

\begin{abstract}
A sensitive, specific and rapid diagnostic test for the detection of Orientia tsutsugamushi, the causative agent of scrub typhus, is necessary to accurately and promptly diagnose patients and ensure that they receive proper treatment. A Loop-Mediated Isothermal Amplification (LAMP) assay targeting the 47-kDa gene of Orientia tsutsugamushi was developed. The LAMP assay was capable of detecting eleven different strains of Orientia at levels comparable to that of the quantitative PCR based method of detection. Ten patient specimens, confirmed to be positive for Orientia by two different PCR methods, were tested and nine out of ten were determined to be positive by LAMP. In terms of specificity, the assay was able to differentiate between Orientia and other phylogenetically similar bacteria as well as mouse and human host DNA. In addition to being sensitive and specific, the LAMP reaction was completed in 1 hour, demonstrating that it is a highly time-efficient method of diagnosing scrub typhus.
\end{abstract}

\section{Introduction}

Scrub typhus, a febrile illness caused by Orientia tsutsugamushi, is endemic to the Asia-Pacific region. The bacteria are transmitted to humans by the bite of larval trombiculid mites, commonly known as chiggers [1]. Approximately 1 million people are infected annually with many more at risk of acquiring infection [2]. The disease can account for up to $23 \%$ of febrile illnesses in the endemic region and can cause up to a $35 \%$ mortality rate if left untreated $[3,4]$. Scrub typhus has been re-emerging and occurring in new areas of many countries including Japan, Korea, Sri Lanka, China, Maldives, India, Palau, Malaysia, Taiwan, and Australia [5-14] and evidence of antibiotic resistance has been shown $[15,16]$. More recently, a new species of Orientia, Orientia chuto was identified in Dubai, where scrub typhus has not previously been described [17]. Furthermore, an endemic scrub typhus-like illness has been documented in Chile recently [18]. These observations clearly show that the disease is gradually expanding to various parts of the world and that there is an immediate need for an accurate method of detection that can cover a broad range of Orientia strains in different geographic regions.

The symptoms of scrub typhus, including fever, headache, and rash, are similar to those of other febrile diseases, making it difficult to differentiate between infections. In order to effectively deliver treatment to patients it is crucial for clinicians to have the ability to accurately distinguish between these symptomatically similar infections. Several methods are currently available for diagnosing scrub typhus including nucleic acid based detection and serological techniques. Although these methods are useful, they have limitations, especially in areas without sufficient resources. For example, quantitative real-time PCR or nested PCR targeting different genes, including the $56 \mathrm{kDa}$ [19], 47kDa [20], GroEL [21], requires the use of an expensive, complex thermocycler which can be very difficult if not impossible to obtain and maintain in resource poor settings. Serological techniques are limited because they require a wait period for antibodies to be produced to a detectable level, and sometimes even healthy individuals in endemic areas can test positive due to the persistence of antibodies from previous scrub typhus infections [22]. Furthermore, the requirement for consistent and high quality antigen preparation has been an obstacle for the development of these assays. Therefore, there is an urgent need to develop a sensitive and specific assay that can be used in endemic areas where there are limited resources.

A Loop-Mediated Isothermal Amplification (LAMP) method has been extensively used as an alternative nucleic acid test to PCR or realtime PCR, and has demonstrated similar sensitivity levels for various infectious diseases [23-29]. Here we describe the detection of Orientia tsutsugamushi DNA by targeting the $47-\mathrm{kD}$ a gene using LAMP. The $47-$ $\mathrm{kDa}$ gene sequence is highly conserved with $>97 \%$ identity in 25 different strains of Orientia [30]. Therefore, the gene is an excellent target in achieving broad phylogenetic and geographic coverage. The LAMP assay was shown to be capable of detecting multiple strains of Orientia with high sensitivity levels similar to that obtained by real-time PCR using the same target. The presence of excess host genomic DNA or inclusion of near-neighbor rickettsial DNA did not interfere with the assay. Furthermore, when only near-neighbor rickettsial DNA was included in the reaction without Orientia template, no false positives were seen. In addition, the LAMP assay successfully identified PCRconfirmed, positive patient samples as positive for Orientia. These results suggest that the LAMP assay can substitute real-time PCR for the sensitive detection of Orientia DNA in resource-limited endemic areas.

*Corresponding author: Chien-Chung Chao, Viral and Rickettsial Diseases Department, Infectious Diseases Directorate, Naval Medical Research Center Silver Spring, MD, USA, E-mail: chien-chung.chao@med.navy.mil

Received May 07, 2012; Accepted June 05, 2012; Published June 12, 2012

Citation: Huber E, Ji D, Howell L, Zhang Z, Chen HW, et al. (2012) Loop-Mediated Isothermal Amplification Assay Targeting the 47-Kda Gene of Orientia tsutsugamushi: A Rapid and Sensitive Alternative to Real-Time PCR. J Med Microb Diagn 1:112. doi:10.4172/2161-0703.1000112

Copyright: (C) 2012 Huber E, et al. This is an open-access article distributed under the terms of the Creative Commons Attribution License, which permits unrestricted use, distribution, and reproduction in any medium, provided the original author and source are credited. 
Citation: Huber E, Ji D, Howell L, Zhang Z, Chen HW, et al. (2012) Loop-Mediated Isothermal Amplification Assay Targeting the 47-Kda Gene of Orientia tsutsugamushi: A Rapid and Sensitive Alternative to Real-Time PCR. J Med Microb Diagn 1:112. doi:10.4172/2161-0703.1000112

\section{Materials and Methods}

\section{Design of primers}

Oligonucleotide primers used for the LAMP and quantitative PCR assays were designed based on the $47 \mathrm{kDa}$ gene sequence from the Karp strain of O. tsutsugamushi. Two sets of primers, each consisting of two outer (F3 and B3) and two inner (FIP and BIP) primers, were used for the LAMP assay. All LAMP primers were designed using PrimerExplorer V4 (http://primerexplorer.jp/e/). The primers used for the quantitative PCR assay were designed based on previously described assays $[21,29]$. Two bases were changed from the reverse primer described by Paris et al. [29] to match the Karp strain sequence. All primers were synthesized by Eurofins MWG Operon (Huntsville, AL) and are described in Table 1.

\section{Plasmid and genomic DNA template}

The $47 \mathrm{kDa}$ gene sequence of $O$. tsutsugamushi Karp strain was cloned into a VR1012 vector, the closed circular plasmid was purified using standard Qiagen plasmid mini kit following manufacturer's instruction. The pure plasmid was quantified spectrophotometrically and used as a standard to determine the best primer combination used in the LAMP assay. The optimized assay was then evaluated using genomic DNA material for sensitivity and specificity reported in the results. The genomic DNA template from multiple strains of O. tsutsugamushi including Karp, Gilliam, Kato, TA763, TH1811, TH1812, TH1814, AFC-1, Garton, Ikeda, and MAK119 were used as template in both LAMP and quantitative-PCR as described below. In addition to pure genomic DNA, genomic DNA in the presence of an abundance of nonspecific mouse or human DNA was also used. All genomic DNA was extracted as previously described [31]. Due to the limited amounts of starting materials (i.e., genomic DNA), select strains were amplified using the Illustra GenomiPhiV2 DNA Amplification Kit according to the manufacturer's instructions (GE Healthcare, Waukesha, WI) prior to performing the LAMP reaction [32].

\section{Loop-mediated isothermal amplification reaction}

LAMP reactions were carried out as described previously [33]. Briefly, the reactions were a total volume of $25 \mu \mathrm{L}$ containing $1.6 \mu \mathrm{M}$ of each FIP and BIP primer, $0.4 \mu \mathrm{M}$ of each F3 and B3 primer, $20 \mathrm{mM}$

\begin{tabular}{|c|c|c|c|}
\hline Primer Name & $\begin{array}{l}\text { Length } \\
\text { (bp) }\end{array}$ & $\operatorname{Tm}\left({ }^{\circ} \mathrm{C}\right)$ & Sequence 5'- 3' \\
\hline \multicolumn{4}{|l|}{ LAMP } \\
\hline \multicolumn{4}{|c|}{ Primer Set 1 (\#3) } \\
\hline F3 & 22 & 58.9 & CTATTCATATGGGTAGCTTTGG \\
\hline B3 & 25 & 58.0 & CCTAAATTCTCATTTAATTCTGGAG \\
\hline FIP & 51 & 69.6 & $\begin{array}{l}\text { AGCAAAACTTATGCCTGAGTAAGAT-TTTT- } \\
\text { GACCGATGTTTAATCTTGAAGG }\end{array}$ \\
\hline BIP & 50 & 69.7 & $\begin{array}{l}\text { CTGTACTTGAAGCAGTTGAATGCT-TTTTA- } \\
\text { CATTTAACATACCACGACGAA }\end{array}$ \\
\hline \multicolumn{4}{|c|}{ Primer Set 2 (\#5) } \\
\hline F3 & 24 & 57.7 & TGAAGTTATAAAAGAAGGATCTGC \\
\hline B3 & 23 & 57.4 & GCTTTGATCATTACTTTGTTGCT \\
\hline FIP & 47 & 70.0 & $\begin{array}{l}\text { ACCTGTAAATCTCTTCCTGTTTTGAT-TTTT- } \\
\text { GGAATTGCTCCTGGAGA }\end{array}$ \\
\hline BIP & 49 & 70.6 & $\begin{array}{l}\text { CTGAAAGAGAAGTTGAGCTTTTACG-TTT- } \\
\text { TACTATCCTCACCTTTGTTGG }\end{array}$ \\
\hline \multicolumn{4}{|l|}{ qPCR } \\
\hline $47 \mathrm{kDa}-\mathrm{F}$ & 28 & 58.8 & AACTGATTTTATTCAAACTAATGCTGCT \\
\hline $47 \mathrm{kDa}-\mathrm{R}$ & 30 & 63.3 & TATGCCTGAGTAAGATACGTGAATGGAATT \\
\hline
\end{tabular}

Tris- $\mathrm{HCl}$ ( $\mathrm{pH}$ 8.8), $10 \mathrm{mM} \mathrm{KCl}, 8 \mathrm{mM} \mathrm{MgSO}_{4}, 10 \mathrm{mM}\left(\mathrm{NH}_{4}\right)_{2} \mathrm{SO}_{4}, 0.1 \%$ Triton X-100, 0.8 M betaine (Sigma-Aldrich, St Louis, MO), $1.4 \mathrm{mM}$ dNTP mixture (New England Biolabs, Beverly, MA), 8U Bst DNA polymerase (New England Biolabs, Beverly, MA), and DNA template. The reaction mixture, excluding Bst enzyme, was heated to $95^{\circ} \mathrm{C}$ for $5 \mathrm{~min}$ to denature the DNA template and then incubated on ice for $5 \mathrm{~min}$. After denaturation of the DNA, the Bst DNA polymerase was added to the reaction mixture and the mixture was incubated at $60^{\circ} \mathrm{C}$ for 60 min. Each reaction was terminated by adding $5 \mu \mathrm{L}$ of $10 \mathrm{X}$ BlueJuice ${ }^{\mathrm{mm}}$ (Invitrogen, Carlsbad, CA). The reaction product was electrophoresed on a $\%$ agarose gel stained with a 1:10,000 dilution of GelRed (Phenix Research Products, Asheville, NC) to visualize results.

\section{$\mathrm{kDa}$ - based quantitative PCR}

Quantitative PCR was performed to compare and to confirm the sensitivity of the LAMP assay. The 7500 Fast Real-time PCR System (Applied Biosystems, Foster City, CA) was used to perform the qPCR reactions and analyze the results. Primers designed against the $47-\mathrm{kDa}$ gene sequence of the Karp strain of Orientia, described in previous studies were used (Table 1) [20]. The total volume of each reaction was $20 \mu \mathrm{L}$. Each reaction mixture contained $6 \mu \mathrm{M}$ of the forward primer, 6 $\mu \mathrm{M}$ of the reverse primer, 1X RT2 SYBR Green qPCR Mastermix (SABiosciences, Frederick, MD), and DNA template. An initial 5 minute activation step at $95^{\circ} \mathrm{C}$ was followed by 40 cycles of $95^{\circ} \mathrm{C}$ for 10 seconds, $60^{\circ} \mathrm{C}$ for 30 seconds, and a melting curve determination cycle.

\section{Results}

The LAMP assay targeting the $47 \mathrm{kDa}$ gene sequence of $O$. tsutsugamushi demonstrated high sensitivity and specificity. The sensitivity of the assay was first determined using plasmid DNA and pure Karp genomic DNA. The assay was able to detect down to 20 copies for both plasmid and genomic DNA template (data not shown). After the initial determination of sensitivity, 10 other strains of Orientia were tested to demonstrate the ability of the assay to detect a broad range of isolates. These are representative strains from different geographic areas within the Orientia triangle [34]. The LAMP assay was capable of detecting all other tested strains of Orientia, which included Kato, Gilliam, TA763, TH1811, TH1812, TH1814, AFC-1, Garton, Ikeda, and MAK119, with similar sensitivity. The sensitivity of the LAMP assay was comparable to that of qPCR (Table 2). Among the eleven strains tested, the detection limit for LAMP compared to QPCR was the same or lower for five strains. Among the remaining six strains, LAMP was able to detect between 16-50 copies/reaction.

DNA was extracted from the liver/spleen homogenate of mice challenged with different strains of $O$. tsutsugamushi in order to test the

\begin{tabular}{|l|l|l|}
\hline Strain of Orientia & qPCR detection limit & LAMP detection limit \\
\hline Karp (WGA) & 11 & 17 \\
\hline Gilliam & & \\
\hline Kato & 25 & 50 \\
\hline TA763 & 13 & 28 \\
\hline TH1811 & 11 & 43 \\
\hline TH1812 & 4 & 4 \\
\hline TH1814 & 20 & 20 \\
\hline AFC-1 & 1 & 1 \\
\hline Garton $($ WGA $)$ & 5 & 5 \\
\hline MAK119 & 1 & 16 \\
\hline Ikeda & 2 & 20 \\
\hline
\end{tabular}

Table 2: Detection limit of the LAMP assay compared to quantitative PCR. 
Citation: Huber E, Ji D, Howell L, Zhang Z, Chen HW, et al. (2012) Loop-Mediated Isothermal Amplification Assay Targeting the 47-Kda Gene of Orientia tsutsugamushi: A Rapid and Sensitive Alternative to Real-Time PCR. J Med Microb Diagn 1:112. doi:10.4172/2161-0703.1000112

$\begin{array}{llllll}1 & 2 & 3 & 4 & 5 & 6\end{array}$

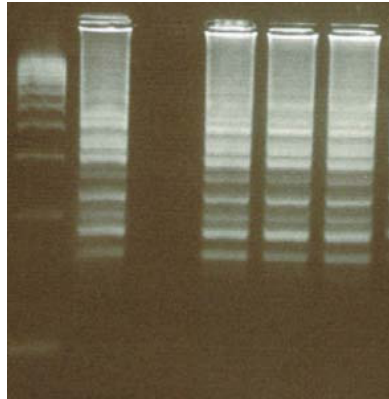

Figure 1: LAMP assay performed using total DNA purified from mouse liver/ spleen homogenate, known to contain the Karp strain of Orientia. Lane 1: 100bp DNA ladder, Lane 2: positive control, Lane 3: negative control, Lane 4-6: products from LAMP reaction containing different copies (lane 4-6 has $20,000,2,000$, and 200 copies, respectively) of Orientia DNA and an overabundance $(1,000$ fold excess) of mouse DNA

\begin{tabular}{|l|l|l|l|}
\hline Sample & 47 kDa gene (accession \#) & Geographic location & Diagnosis* $^{*}$ (strain) \\
\hline L1 & + (L31934) & New Guinea & ST (KARP) \\
\hline L2 & $+($ L31933) & Burma & ST (GILLIAM) ${ }^{2}$ \\
\hline L3 & $+($ L31933) & Burma & ST (GILLIAM) \\
\hline L4 & $+($ L31934) & New Guinea & ST (KARP) \\
\hline L5 & $+($ L31934) & New Guinea & ST (KARP) \\
\hline L6 & $+($ L11697) & Japan & ST (KATO) ${ }^{3}$ \\
\hline L7 & + (HM595490) & Thailand & ST (TA763) \\
\hline L8 & $-($ L11697) & Japan & ST (KATO) \\
\hline L9 & $+($ L11697) & Japan & ST (KATO) \\
\hline L10 & $+($ L31934) & New Guinea & ST (KARP) \\
\hline L11 & $(-)$ & & MT \\
\hline L12 & $(-)$ & & MT \\
\hline L13 & $(-)$ & & LEPTO. \\
\hline L14 & $(-)$ & & LEPTO. \\
\hline L15 & $(+)$ & & MALARIA(PF) \\
\hline L16 & $(-)$ & & MALARIA(PF) \\
\hline L17 & $(-)$ & & DENGUE \\
\hline L18 & $(-)$ & & DENGUE \\
\hline L19 & $(-)$ & HEALTHY \\
\hline L20 & $(+)$ & HEALTHY \\
\hline
\end{tabular}

*. ST: scrub typhus; MT: murine typhus.

${ }^{1}$,Kim,I.J., Kim,I.S., Choi,I.H., Chang,W.H. and Choi,M.S.

Direct deposited to NCBI database, Apr 18, 1994.

2.Kim I.J. Direct deposited to NCBI database, Mar 11, 1996

${ }^{3}$.Kim I.J. Direct deposited to NCBI database, Mar 11, 1996.

4. Jiang,J., Newton,P.N., Phetsouvanh,R., Izzard,L., Stenos,J., Graves,S.R., Hu,X., Wallqvist,A., Ge,H., Day,N.P.J., Richards,A.L.,Blacksell,S.D., Paris,D.H. and Aukkanit,N. Direct deposited to NCBI database Jul 25, 2011.

Table 3: Evaluation of LAMP assay using the $47 \mathrm{kDa}$ gene as the target with PCR confirmed positive and negative samples.

ability of the LAMP assay to detect Orientia in the presence of host DNA (at 1 Orientia DNA/1000 host DNA). The assay was able to detect the target DNA despite the abundance of mouse DNA in the sample (Figure 1). Additionally, in order to simulate the use of this assay in a true clinical setting, the LAMP assay was performed using DNA extracted from patient serum that was determined by two well-validated PCR analyses targeting $16 \mathrm{~s}$ rRNA and the $56 \mathrm{kDa}$ gene to be positive or negative for O. tsutsugamushi $[35,36]$. These DNA materials were kindly provided to us by Dr. Yupin Suputtamongkol (Siriraj Hospital, Mahidol University, Bangkok, Thailand). A total of 20 samples were tested, ten positive and ten negative. Of the ten positive patient samples that were
A)

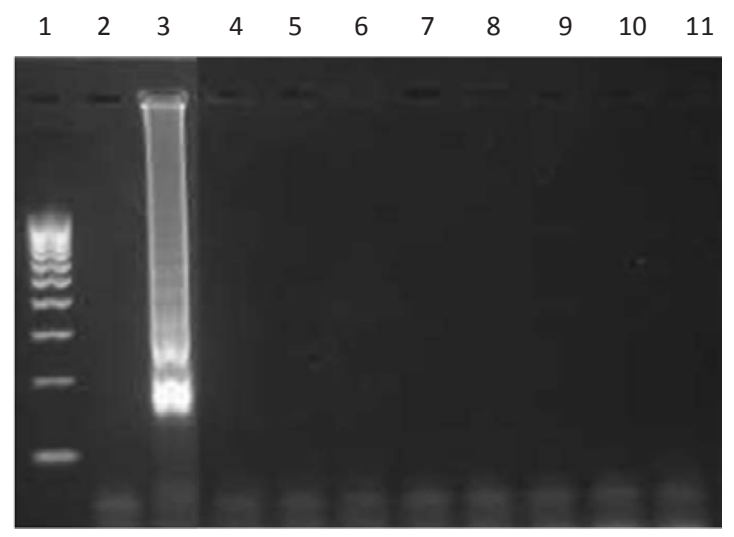

B)

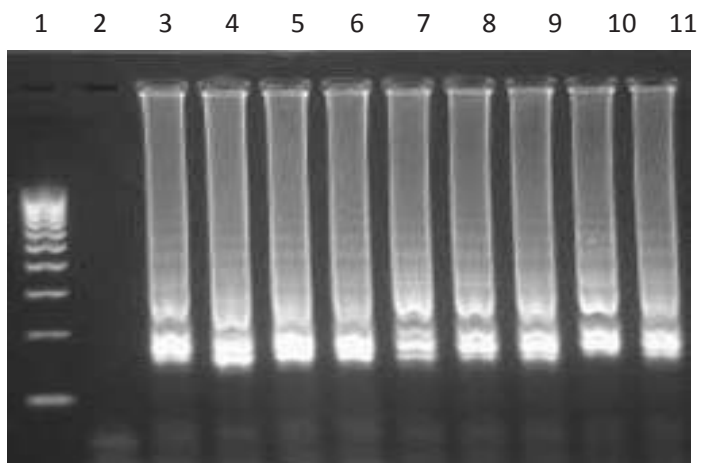

Figure 2: Demonstration of specificity of the LAMP assay. A) Lane 1: 100bp ladder, Lane 2: negative control, Lane 3: positive control, Lane 4-11: products from LAMP reactions containing genomic DNA from bacteria which are phylogenetically similar to Orientia including $R$. conorii $R$. prowazakii, $R$. belli, $R$. rickettsii, $R$. typhi, $R$. felli, C. burnetii, and L. interrogans, respectively. B) LAMP assay performed using a combination of Orientia genomic DNA and other bacterial genomic DNA. Lane 1: 100 bp ladder, Lane 2: negative control, Lane 3: positive control, Lane 4-11: O.tsu + R. conorii, O.tsu + R. prowazakii, O.tsu + R. belli, O.tsu + R. rickettsii, O.tsu + R. typhi, O.tsu + R. felli, O.tsu + C. burnetii, and O.tsu + L. interrogans, respectively.

confirmed positive by PCR, the LAMP assay detected nine out of the ten as being positive. Among these samples the Karp, Gilliam, Kato, and TA763 strains of Orientia were represented. The false negative was determined to be of the Kato strain of Orientia. Of the ten PCR-confirmed negative patient samples, the LAMP assay determined eight out of the ten samples to be negative. The other two samples showed a positive signal with a similar banding pattern to the Orientia positive samples (Table 3).

The specificity of the LAMP assay was also demonstrated by using purified DNA from bacteria that are phylogenetically close to Orientia as the template. LAMP reactions containing $R$. conorii, $R$. prowazakii, $R$. belli, R. rickettsii, R. typhi, $R$. felis, C. burnetii, or L. interrogans DNA as template all tested negative (Figure 2A). Addition of the Karp strain of Orientia at 1/10 of DNA copy of other DNA resulted in positive results for all reactions, indicating that the assay is specifically detecting Orientia and sensitive enough to detect the desired target in 
the presence of other phylogenetically close genomic DNA (Figure 2B).

\section{Discussion}

The $47 \mathrm{kDa}$ gene sequence was selected as a target for the LAMP assay because it is very conserved among the different strains of Orientia with the exception of $O$. chuto [18]. As stated previously, the $47 \mathrm{kDa}$ gene has $>97 \%$ identity in 25 different strains of Orientia [5,22]. The ability of this LAMP assay to cover a broad range of isolates was shown. This indicates that the assay could be utilized in various geographic settings. The strains able to be detected by the LAMP assay are endemic to multiple countries including Thailand, Taiwan, Japan, New Guinea, Australia and Burma [34].

During the optimization process of the development of the LAMP assay, several sets of primers were screened individually and in combination. Combining two sets of primers without loop primers was proved to be more sensitive in amplifying the target DNA than using one set alone. It should be noted that while an incorrect loop primer was mistakenly added to LAMP reactions during some trials with the Karp and Gilliam strains initially, follow up experiments conducted confirmed that this error did not interfere with the reaction (the LAMP detection limit with the incorrect loop primer was 6 copies and 55 copies for Karp and Gilliam, respectively, Table 2). This is consistent with the notion that the primary function of loop primers is to accelerate the reaction and not to improve sensitivity or specificity [37]. To further increase the sensitivity of the assay, a TTTT-linker was added to the inner primer sequences, which allows the primers to have more physical flexibility in binding to the target DNA. Temperatures ranging between 59 and $65^{\circ} \mathrm{C}$ were tested to determine the optimal incubation temperature for the LAMP reaction. Sixty degrees was the temperature at which the assay performed with the highest sensitivity. Also in an effort to increase the sensitivity of the assay, the DNA template was denatured to increase the amount of exposed single stranded DNA to which the primers could bind to. In addition to the method used, denaturing the DNA after adding it to the reaction mixture, a second method was tested in which the DNA was denatured prior to adding it to the reaction mixture. The latter method was found to be less efficient as the method that was used for this study. The primers being present in the mixture when the DNA is denatured likely increases the amount of primer that will anneal to the DNA.

Whole genome amplification (WGA) was performed using the Illustra GenomiPhiV2 DNA Amplification Kit with the Karp and Garton strains of Orientia to obtain more genomic material prior to running the LAMP assay. This was due to the limited amount of pure DNA material from these two strains. Kato et al. [32] had shown that WGA of low amounts of rickettsia DNA in the presence or absence of host DNA material could lead to an increased amount of rickettsial DNA available as template for downstream qPCR amplification. The WGA reaction used in this study also showed amplification of Orientia DNA and subsequent detection by LAMP and qPCR. It was not clear whether there was a difference in efficiency of amplification of Orientia DNA with or without the presence of host DNA material. Nevertheless, it appeared that both genomic materials were amplified when WGA was applied. The use of WGA posed an uncertainty as to whether the 47 gene sequence would be over-amplified during this procedure. Therefore, quantitative PCR was performed and it was confirmed that the 47 gene sequence was not over-amplified and that the amplification process did not alter the genomic DNA or the interpretation of the results (data not shown). This observation suggests that WGA can be incorporated in the sample preparation step to increase the amount of target DNA for a subsequent PCR or real-time PCR assay. Alternatively, WGA can be applied to hard-to-get DNA samples in order to preserve the precious material for downstream applications.

The one confirmed-positive clinical sample that was not detected as positive by LAMP was characterized and found to be of the Kato strain. This is the only one among the three Kato strain positives that was not detected by LAMP. It is not clear why this particular Kato strain infected patient sample was not detected by LAMP. One possibility is that there was a low amount of DNA extracted from this sample, or that the target region of the LAMP primers, the $47 \mathrm{kDa}$ gene sequence, was not available in the purified DNA material, as the PCR assays originally determining positive or negative status targeted the $56 \mathrm{kDa}$ and $16 \mathrm{~s}$ rRNA genes. We performed additional experiments to confirm the presence of the $56 \mathrm{kDa}$ gene using nest PCR [30] and were not able to detect the gene. Even after whole genome amplification of the original DNA material followed by nest PCR, we were not able to detect the $56 \mathrm{kDa}$ gene. The WGA amplified material was not positive by LMAP assay either (data not shown). Therefore, it is possible that this sample was misdiagnosed as scrub typhus patient serum.

The assay demonstrated high specificity when tested with DNA template that was phylogenetically close to that of Orientia. However, two out of ten clinical samples that were PCR-confirmed negatives were tested positive by the LAMP assay. Given that the banding pattern for these two samples was similar to that of Orientia positive, it is reasonable to think that these samples were positive due to contamination by Orientia genomic material and not because the primers were nonspecifically binding to the host or other DNA. Further experiments to demonstrate the specificity of the current LAMP assay by eliminating contamination are desirable and more clinical samples shall be tested to evaluate the clinical usage of the assay. Two recent publications by Blacksell et al. [38] and Paris et al. [39] have examined the clinical usage of the LAMP assay targeting the groEL gene individually or in combination with serological assays using well-characterized scrub typhus patient samples. It was concluded that the use of either a serological assay or LAMP assay alone would not provide desired levels of sensitivity and specificity. The combination of both serological assay and nucleic acid detection covering a complete time frame during the progression of disease is the best clinical practice for accurate diagnosis of the disease. However, the cost of performing both serological assay and nucleic acid test (i.e. qPCR or PCR) in low resource, endemic areas can be substantial. With the development of an immunochromatographic test for serological testing and the less expensive, qPCR comparable LAMP assay, it is possible to perform both assays in parallel at an affordable price to provide timely and accurate diagnosis for proper treatment.

\section{Acknowledgement}

The authors wish to thank Dr. Yupin Suputtamongkol and her associates to provide DNA extracted from clinical samples. These samples were confirmed as positive or negative using PCR. This work was supported by Work Unit Number (WUN) 6000.RAD1.J.A0310. The opinions and assertions contained herein are the private ones of the authors and are not to be construed as official or as reflecting the views of the Department of the Navy, the Naval service at large, the Department of Defense, or the U. S. Government. Authors Chien-Chung Chao and Wei-Me Ching are employees of the U. S. Government. This work was prepared as part of official duties. Title 17 U.S.C. $\$ 105$ provides that 'Copyright protection under this title is not available for any work of the United States Government.' Title 17 U.S.C. $\S 101$ defines a U.S. Government work as a work prepared by employee of the U.S Government as part of that person's official duties.

\section{References}

1. Watt G, Parola P (2003) Scrub typhus and tropical rickettsioses. Curr Opin Infect Dis 16: 429-436. 
Citation: Huber E, Ji D, Howell L, Zhang Z, Chen HW, et al. (2012) Loop-Mediated Isothermal Amplification Assay Targeting the 47-Kda Gene of Orientia tsutsugamushi: A Rapid and Sensitive Alternative to Real-Time PCR. J Med Microb Diagn 1:112. doi:10.4172/2161-0703.1000112

2. Kuo CC, Huang JL, Ko CY, Lee PF, Wang HC (2011) Spatial analysis of scrub typhus infection and its association with environmental and socioeconomic factors in Taiwan. Acta Trop 120: 52-58.

3. Brown GW, Robinson DM, Huxsoll DL, Ng TS, Lim KJ (1976) Scrub typhus: a common cause of illness in indigenous populations. Trans $\mathrm{R}$ Soc Trop Med Hyg 70: 444-448.

4. Brown GW, Saunders JP, Singh S, Huxsoll DL, Shirai A (1978) Single dose doxycycline therapy for scrub typhus. Trans R Soc Trop Med Hyg 72: 412-416.

5. Kumar K, Saxena VK, Thomas TG, Lal S (2004) Outbreak investigation of scrub Typhus in Himachal Pradesh (India). J Commun Dis 36: 277-283.

6. Chang WH (1995) Current status of tsutsugamushi disease in Korea. J Korean Med Sci 10: 227-238.

7. Jiang J, Marienau KJ, May LA, Beecham HJ 3rd, Wilkinson R, et al. (2003) Laboratory diagnosis of two scrub typhus outbreaks at Camp Fuji, Japan in 2000 and 2001 by enzyme-linked immunosorbent assay, rapid flow assay, and Western blot assay using outer membrane $56-\mathrm{kD}$ recombinant proteins. Am J Trop Med Hyg 69: 60-66.

8. Sharma A, Mahajan S, Gupta ML, Kanga A, Sharma V (2005) Investigation of an outbreak of scrub typhus in the himalayan region of India. Jpn J Infect Dis 58: $208-210$

9. Wang S, Huang J, Peng G, Jiang P, Zheng N, et al. (2002) Natural foci of tsutsugamushi disease in the Nan Peng Lie Islands in China. Chin Med J (Engl) 115: 272-275.

10. Lewis MD, Yousuf AA, Lerdthusnee K, Razee A, Chandranoi K, et al. (2003) Scrub typhus reemergence in the Maldives. Emerg Infect Dis 9: 1638-1641.

11. Durand AM, Kuartei S, Togamae I, Sengebau M, Demma L, et al. (2004) Scrub typhus in the Republic of Palau, Micronesia. Emerg Infect Dis 10: 1838-1840.

12. Tay ST, Kamalanathan M, Rohani MY (2003) Antibody prevalence of Orientia tsutsugamushi, Rickettsia typhi and TT118 spotted fever group rickettsiae among Malaysian blood donors and febrile patients in the urban areas. Southeast Asian J Trop Med Public Health 34: 165-170.

13. Odorico DM, Graves SR, Currie B, Catmull J, Nack Z, et al. (1998) New Orientia tsutsugamushi strain from scrub typhus in Australia. Emerg Infect Dis 4: 641644.

14. Lee YS, Wang PH, Tseng SJ, Ko CF, Teng HJ (2006) Epidemiology of scrub typhus in eastern Taiwan, 2000-2004. Jpn J Infect Dis 59: 235-238.

15. Watt G, Chouriyagune C, Ruangweerayud R, Watcharapichat $P$, Phulsuksombati $D$, et al. (1996) Scrub typhus infections poorly responsive to antibiotics in northern Thailand. Lancet 348: 86-89.

16. Mathai E, Rolain JM, Verghese GM, Abraham OC, Mathai D, et al. (2003) Outbreak of scrub typhus in southern India during the cooler months. Ann N Y Acad Sci 990: 359-364.

17. Izzard L, Fuller A, Blacksell SD, Paris DH, Richards AL, et al. (2010) Isolation of a novel Orientia species (O. chuto sp. nov.) from a patient infected in Dubai. $\mathrm{J}$ Clin Microbiol 48: 4404-4409.

18. Balcells ME, Rabagliati R, García P, Poggi H, Oddó D, et al. (2011) Endemic scrub typhus-like illness, Chile. Emerg Infect Dis 17: 1659-1663.

19. Prakash JA, Reller ME, Barat N, Dumler JS (2009) Assessment of a quantitative multiplex 5' nuclease real-time PCR for spotted fever and typhus group rickettsioses and Orientia tsutsugamushi. Clin Microbiol Infect 15 Suppl 2: 292293.

20. Jiang J, Chan TC, Temenak JJ, Dasch GA, Ching WM, et al. (2004) Development of a quantitative real-time polymerase chain reaction assay specific for Orientia tsutsugamushi. Am J Trop Med Hyg 70: 351-356.

21. Paris DH, Aukkanit N, Jenjaroen K, Blacksell SD, Day NP (2009) A highly sensitive quantitative real-time PCR assay based on the groEL gene of contemporary 339 Thai strains of Orientia tsutsugamushi. Clin Microbiol Infect 15: 488- 495.

22. Eamsila C, Singsawat P, Duangvaraporn A, Strickman D (1996) Antibodies to Orientia tsutsugamushi in Thai soldiers. Am J Trop Med Hyg 55: 556-559.

23. Ihira M, Akimoto S, Miyake F, Fujita A, Sugata K, et al. (2007) Direct detection of human herpesvirus 6 DNA in serum by the loop-mediated isothermal amplification method. J Clin Virol 39: 22-26.

24. Iwamoto T, Sonobe T, Hayashi K (2003) Loop-mediated isothermal amplifica- tion for direct detection of Mycobacterium tuberculosis complex, M. avium, and M. intracellulare in sputum samples. J Clin Microbiol 41: 2616-2622.

25. Iwata S, Shibata Y, Kawada J, Hara S, Nishiyama Y, et al. (2006) Rapid detection of Epstein-Barr virus DNA by loop-mediated isothermal amplification method. J Clin Virol 37: 128-133.

26. Higashimoto $Y$, Ihira M, Ohta A, Inoue S, Usui C et al (2008) Discriminating between varicella-zoster virus vaccine and wild-type strains by loop-mediated isothermal amplification. J Clin Microbiol 46: 2665-2670.

27. Miyagawa J, Maeda H, Murauchi T, Kokeguchi S, Yamabe K, et al. (2008) Rapid and simple detection of eight major periodontal pathogens by the loopmediated isothermal amplification method. FEMS Immunol Med Microbiol 53 314-321.

28. Peyrefitte CN, Boubis L, Coudrier D, Bouloy M, Grandadam M, et al. (2008) Real-time reverse-transcription loop-mediated isothermal amplification for rapid detection of rift valley Fever virus. J Clin Microbiol 46: 3653-3659.

29. Paris DH, Blacksell SD, Newton PN, Day NP (2008) Simple, rapid and sensitive detection of Orientia tsutsugamushi by loop-isothermal DNA amplification. Trans R Soc Trop Med Hyg 102: 1239-1246.

30. Chen HW, Zhang Z, Huber E, Chao CC, Wang H, et al. (2009) Identification of cross-reactive epitopes on the conserved 47-kilodalton antigen of Orientia tsutsugamushi and human serine protease. Infect Immun 77: 2311-2319.

31. Ge H, Chuang YY, Zhao S, Tong M, Tsai MH, et al. (2004) Comparative genomics of Rickettsia prowazekii Madrid E and Breinl strains. J Bacteriol 186: 556-565.

32. Kato CY, Robison LK, White FH, Eremeeva ME, Dasch GA. Inexpensive and high throughput whole genome amplification for improved characterization of Rickettsia DNAs. 2009 in 23rd American Society for Rickettsiology meeting, Crowne Plaza Resort, Hilton Head Island, South Carolina, USA, Aug 15-18, 2009

33. Notomi T, Okayama H, Masubuchi H, Yonekawa T, Watanabe K, et al. (2000) Loop-mediated isothermal amplification of DNA. Nucleic Acids Res 28: E63.

34. Chao CC, Huber ES, Porter TB, Zhang Z, Ching WM (2011) Analysis of the cross-reactivity of various $56 \mathrm{kDa}$ recombinant protein antigens with serum samples collected after Orientia tsutsugamushi infection by ELISA. Am J Trop Med Hyg 84: 967-972.

35. Sonthayanon P, Chierakul W, Wuthiekanun V, Blacksell SD, Pimda K, et al (2006) Rapid diagnosis of scrub typhus in rural Thailand using polymerase chain reaction. Am J Trop Med Hyg 75: 1099-1102.

36. Wongprompitak P, Anukool W, Wongsawat E, Silpasakorn S, Dyong V, et al. (2011) Broad-coverage molecular epidemiology of Orientia tsutsugamushi in Thailand. Infect Genet Evol.

37. Nagamine K, Hase T, Notomi T (2002) Accelerated reaction by loop-mediated isothermal amplification using loop primers. Mol Cell Probes 16: 223-229.

38. Blacksell SD, Paris DH, Chierakul W. Wuthiekanun V, Teeratakul A, et al. (2012) Prospective evaluation of commercial antibody-based rapid tests in combination with LAMP for the detectin of Orientia tsutsugamushi during the acute phase of scrub typhus infection. Clin Vaccine Immunol 19: 391-395.

39. Paris DH, Blacksell SD, Nawtaisong P, Jenjaroen K, Teeraratkul A, et al. (2011) Diagnostic accuracy of a loop-mediated isothermal PCR assay for detection of Orientia tsutsugamushi during acute scrub typhus infection. PLoS Negl Trop Dis 5: e1307. 